\title{
Lipid metabolism: An alternative Achille's heel in lymphomas?
}

\author{
Pier Paolo Piccaluga ${ }^{1,2 *}$, Axel Visani ${ }^{1}$, Cristiano Peron ${ }^{1}$, Flavia Rigotti ${ }^{1}$, Lorenzo Leoncini ${ }^{3}$ and Giuseppe Visani $^{4}$ \\ ${ }^{1}$ Department of Experimental, Diagnostic, and Experimental Medicine, Bologna University School of Medicine, Italy \\ ${ }^{2}$ Department of Genomics and Personalized Medicine, Euro-Mediterranean Institute of Science and Technology (IEMEST), Italy \\ ${ }^{3}$ Section of Pathology, Department of Medical Biotechnology, University of Siena, Italy \\ ${ }^{4}$ Hematology and Hematopoietic Stem Cell Transplant Center, AORMN, Italy
}

Multiple molecular mechanisms, both intrinsic and extrinsic, converge to alter core cellular metabolism and to provide support for the three basic needs of dividing cells: rapid ATP generation to maintain energy status, increased biosynthesis of macromolecules, and the strict maintenance of an appropriate redox status. To meet these needs, cancer cells acquire alterations to the metabolism of all four major classes of macromolecules: carbohydrates, proteins, lipids and nucleic acids [1]. In these processes the environment plays a key role, as the concentration and availability of the nutrients essential for these different and increased metabolic needs may be highly heterogeneous. Based on that, metabolic modifications in cancer cells have been the object of many attentions by several authors in the last decades.

The oldest, best known and characterized metabolic phenotype present in tumor cells is the Warburg effect [2]. First postulated by Nobel laureate Otto Warburg in 1924, it details the metabolic shift of highly proliferating cells (Warburg's actual first hypothesis considered only neoplastic cells to be interested by the phenomenon, but in time other non-neoplastic cell types were also found to be involved) towards aerobic glycolysis. While under normal circumstances cells adopt glycolysis in anaerobic conditions when the amount of oxygen is insufficient, many tumor cells seem to prefer to adopt glycolysis as their main energetic pathway even when oxygen availability is perfectly normal, thus giving this process the name of aerobic glycolysis. Although apparently baffling, a cell using glycolysis instead of oxidative phosphorylation might have some minor advantages in terms of energy production speed: the first pathway allows in fact cells to produce the same quantity of ATP in a minor amount of time, all this at the expense of efficiency, with oxidative phosphorylation being far better in terms of ATP produced per glucose molecule. Currently, it is postulated that the use of glycolysis byproducts by transformed cells allows to gain biosynthetic advantage. A high flux of substrate through glycolysis, in fact, allows for an effective shunting of carbon to key subsidiary biosynthetic pathways (1). The preferential use of glycolysis even in aerobic conditions ultimately allows the neoplastic cell for an improved synthesis of macromolecules, which is, as noted earlier, one of the key points for cancer cell metabolism [3].

Several factors are involved and contribute to this process, most of which have been found directly or indirectly affected by somatic mutations and other genetic aberrations in cancer cells. One of the most notable examples is probably the PI3K pathway, which, via the downstream effector AKT1, manages to phosphorylate several key glycolytic enzymes and to strongly promote the activity of the kinase mTOR, whose main role is to couple macromolecular biosynthesis and cell growth with nutrient availability [4]. Once activated, it also promotes the activation under normoxic conditions of HypoxiaInducible Factor 1 (HIF1), which in turn cooperates with MYC in the final shift towards the glycolytic phenotype, by amplifying the transcription of glucose membrane transporters and key glycolytic enzymes, while in turn reducing oxidative phosphorylation. MYC also promotes the expression of Pyruvate Kynase M2 [5], which, despite being scarcely effective at promoting glycolysis, grants the tumor a substantial advantage by allowing carbohydrate metabolites to enter other pathways and ultimately generate macromolecules [6]. Several other factors who, on the contrary, work towards creating a balance between glycolysis and oxidative phosphorylation by promoting the latter, are found inactivated in neoplastic cells. Among these are AMPK (whose pathway can still be stimulated thanks to common antidiabetic drugs such as metformin and phenoformin) [7], and, most notably, P53 and OCT1 [1]. Lastly, low-to-medium levels of reactive oxygen species can promote cell proliferation and survival, and the expression of factor such as HIF and VEGF, thus explaining the importance of efficient antioxidant systems such as glutathione and thioredoxin in tumor cells [8].

The study of lipid metabolism in human neoplasms is a relatively new subject. Lipids, especially fatty acids, may play different roles in transformed cells. First of all, neoplastic cells are actively proliferating cells, and thus they need fatty acids for the synthesis of structural lipids [9]. Secondly, the abundance and saturation level of fatty acids determines the activity of signaling proteins that require acylation for their activation, as for example the WNT proteins, which are frequently deregulated in human cancer [10]. Third, various lipids can function as important signaling molecules, like diacylglycerol (DAG), inositol triphosphate (IP3), and phosphatidylinositol $(3,4,5)$-triphosphate (PIP3) [11]. Last, neoplastic cells rely heavily on fatty acid uptake under hypoxic conditions as a means of compensating reduced fatty acid oxidation, while, upon re-oxygenation, they use fatty acids both for energy production and antioxidant defense. Three main lipid-related metabolic processes have been found altered in human malignancies: fatty acid synthesis, fatty acid oxidation, and fatty acid storage and

Correspondence to: Pier Paolo Piccaluga, Department of Experimental, Diagnostic, and Experimental Medicine, Bologna University School of Medicine, via Massarenti 9, 40138, Bologna, Italy, Tel: +390512144043; E-mail: pierpaolo. piccaluga@unibo.it

Received: June 14, 2017; Accepted: June 26, 2017; Published: June 28, 2017 
mobilization. Fatty acid biosynthesis is increased in tumor cells because of the aforementioned reasons: lipids are needed for macromolecular biosynthesis, acylation, signaling, and hypoxia response [9]. Both key enzymes involved in this process and the transcriptional regulators responsible for their expression can be deregulated in human cancers. As far as fatty acid oxidation is concerned, its actual level of involvement in the transformation process is still largely unknown. Noteworthy, the CPT1C isoform of an enzyme linked with fatty acid oxidation, carnitine palmitoyltranferase (CPT), is overexpressed in different human cancer cells. Lipid storage, mobilization and transport also play a key role, as the active fatty acid pool available for cancer cell growth and proliferation can be regulated by diverting fatty acids to storage or by blocking their release from storage. Lastly, the reactivation of fatty acid biosynthesis in cancer changes the overall saturation levels of membrane lipids by increasing the relative amount of saturated and monounsaturated species, while polyunsaturated forms, which are mainly derived from dietary lipids, are reduced. This higher saturation level protects cancer cells from reactive oxygen species (ROS), as saturated membrane lipids are less susceptible to peroxidation.

Based on these premises, different studies tried to evaluate the correlation between lipid metabolism and cancer in various human malignancies. Dysregulation of lipid metabolism, including both synthesis and oxidation, is a common event in breast cancer (12). It has been observed that breast cancers have developed a dependency on the altered expression of several key enzymes including fatty acid synthase (FASN), acetyl-CoA carboxylase, fatty acid binding protein 4 (FABP4), and malonyl-CoA decarboxylase. Phosphatidylcholine plays important roles in rapidly proliferating cancer cells as both a membrane constituent and a source of the signaling molecule DAG. More recent studies demonstrated that MYC expressing triple-negative breast cancer heavily relies on fatty acid oxidation for its metabolic needs, and that the inhibition of this pathway can be used for therapeutic purposes [13]. Similarly, MYC-positive lung cancer shows an increase in phosphatidylinositol and in arachidonate-containing phospholipids when compared to normal lung tissue [14].

Studies on lipid metabolism in haematological malignancies are not completely unheard of: in 2010 Samudio et al. [15] demonstrated how important fatty acids and fatty acid oxidation are for human leukemia cells. In the study, it was shown how etomoxir and ranolazine, two fatty acid oxidation inhibitors, and orlistat, a well-known anti-obesity drug which works as an inhibitor of lipolysis, inhibited the proliferation of neoplastic cells, while sensitizing them to apoptosis induction [15]. In chronic lymphocytic leukemia (CLL) metabolism alterations have been studied by several Authors [16]. Unlike normal B lymphocytes, CLL cells, like adipocytes, store lipids and utilize free fatty acids (FFA) to produce chemical energy. Apparently, none of the known gene mutations occurring in CLL directly affects metabolic pathways, while activation of STAT3 or downregulation of microRNA-125 levels seems to plays a crucial role [16]. Conversely, only very few data concerning lipid metabolism in human lymphomas have been so far published. Particularly, lipid metabolism activation was reported in Burkitt and diffuse large B-cell lymphomas [17]. Furthermore, a recent study showed that fenofibrate associated effects on hepatic lipid metabolism and deprivation of serum lipids are capable to suppress B-cell lymphoma growth in mice [18]. Therefore, the question "might lipid metabolism represent an alternative Achille's heel in human lymphomas?" is cogently asking to be answered.

\section{Acknowledgements}

This work was supported by AIL Pesaro Onlus.

\section{References}

1. Cairns RA, Harris IS, Mak TW (2011) Regulation of cancer cell metabolism. Nat Rev Cancer 11: 85-95. [Crossref]

2. Warburg O, Posener K, Negelein E (1924) Ueber den Stoffwechsel der Tumoren. Biochemische Zeitschrift. 1521924: 319-44.

3. Vander Heiden MG, Cantley LC, Thompson CB (2009) Understanding the Warburg effect: the metabolic requirements of cell proliferation. Science 324: 1029-33. [Crossref]

4. Robey RB, Hay N (2009) Is Akt the "Warburg kinase"?-Akt-energy metabolism interactions and oncogenesis. Semin Cancer Biol 19: 25-31. [Crossref]

5. David CJ, Chen M, Assanah M, Canoll P, Manley JL (2010) HnRNP proteins controlled by c-Myc deregulate pyruvate kinase mRNA splicing in cancer. Nature 463: 364-368. [Crossref]

6. Christofk HR, Vander Heiden MG, Harris MH, Ramanathan A, Gerszten RE, et al. (2008) The M2 splice isoform of pyruvate kinase is important for cancer metabolism and tumour growth. Nature 452: 230-233. [Crossref]

7. Lei Y, Yi Y, Liu Y, Liu X, Keller ET, et al. (2017) Metformin targets multiple signaling pathways in cancer. Chin J Cancer 36: 17. [Crossref]

8. Gao P, Zhang H, Dinavahi R, Li F, Xiang Y, et al. (2007) HIF-dependent antitumorigenic effect of antioxidants in vivo. Cancer Cell 12: 230-238. [Crossref]

9. Röhrig F, Schulze A (2016) The multifaceted roles of fatty acid synthesis in cancer. Nat Rev Cancer 16: 732-749. [Crossref]

10. Nile AH, Hannoush RN (2016) Fatty acylation of Wnt proteins. Nat Chem Biol 12 60-69. [Crossref]

11. Vanhaesebroeck B, Stephens L, Hawkins P (2012) PI3K signalling: the path to discovery and understanding. Nat Rev Mol Cell Biol 13: 195-203. [Crossref]

12. Borgan E, Sitter B, Lingjaerde OC, Johnsen H, Lundgren S, et al. (2010) Merging transcriptomics and metabolomics--advances in breast cancer profiling. BMC Cancer 10: 628. [Crossref]

13. Camarda R, Zhou AY, Kohnz RA, Balakrishnan S, Mahieu C, et al. (2016) Inhibition of fatty acid oxidation as a therapy for MYC-overexpressing triplenegative breast cancer. Nat Med 22: 427-432. [Crossref]

14. Hall Z, Ament Z, Wilson CH, Burkhart DL, Ashmore T, et al. (2016) Myc Expression Drives Aberrant Lipid Metabolism in Lung Cancer. Cancer Res 76: 4608-4618. [Crossref]

15. Samudio I, Harmancey R, Fiegl M, Kantarjian H, Konopleva M, et al. (2010) Pharmacologic inhibition of fatty acid oxidation sensitizes human leukemia cells to apoptosis induction. J Clin Invest 120: 142-156. [Crossref]

16. Rozovski U, Hazan-Halevy I, Barzilai M, Keating MJ, Estrov Z (2015) Metabolism pathways in chronic lymphocytic leukemia, Leuk Lymphoma 57: 758-765. [Crossref]

17. Ambrosio MR, Piccaluga PP, Ponzoni M, Rocca BJ, Malagnino V, et al. (2012) The Alteration of Lipid Metabolism in Burkitt Lymphoma Identifies a Novel Marker: Adipophilin. PLoS One 7: e44315. Published online 2012 Aug 31. doi: 10.1371/ journal.pone.0044315. [Crossref]

18. Huang J, Das SK, Jha P, Al Zoughbi W, Schauer S, et al. (2013) The PPARa agonis fenofibrate suppresses B-cell lymphoma in mice by modulating lipid metabolism. Biochim Biophys Acta 1831: 1555-1565. [Crossref]

Copyright: (C2017 Piccaluga PP. This is an open-access article distributed under the terms of the Creative Commons Attribution License, which permits unrestricted use, distribution, and reproduction in any medium, provided the original author and source are credited. 